

\section{Chronic anemia and non-ST elevation acute coronary syndrome - double jeopardy}

Phillip Freeman ${ }^{\mathrm{a}}$, James Berrill ${ }^{\mathrm{b}}$, John Green ${ }^{\mathrm{c}}$ and Richard Anderson ${ }^{\mathrm{b}}$

\section{Introduction}

The clinical presentation of patients with a non-ST-elevation acute coronary syndrome (NSTEACS) and coexisting anemia is a frequent clinical situation. Gastroenterology teams may be reluctant to perform invasive endoscopic diagnostic tests or treatments at such an early stage after high risk NSTEACS, and cardiology teams may be unwilling to perform invasive investigation and treatment until the etiology of the anemia is better understood. It presents a common and complex dilemma for medical teams, and can result in prolonged hospital admission and increased patient anxiety; if managed inappropriately it can have an adverse effect on patient outcomes.

\section{Scope of review}

We outline the priorities from cardiology and gastroenterology perspectives, resolve some of the misconceptions, and use the evidence available to suggest strategies for optimizing management of these patients. The dual presentation of acute active gastrointestinal (GI) blood loss together with an acute coronary syndrome (NSTEACS or STEMI [ST elevation myocardial infarction]) is outside the remit of this review but has been previously reviewed by our group ${ }^{1}$. This review covers NSTEACS together with chronic or sub-acute anemia, anemia that cannot be accounted for by chronic kidney disease ${ }^{2}$, heart failure ${ }^{3}$, or inflammatory disorders. Identification of a potential GI etiology of anemia is important as it allows adjustment to both GI and CV investigations and treatments that can reduce the likelihood and impact of bleeding complications.

\section{Non-ST-elevation ACS in an expanding group of patients}

In the United States, NSTEACS affects $>625,000$ patients annually, or three quarters of all patients with acute coronary syndrome (ACS) ${ }^{4}$. In the new ACC/AHA guidelines for management of NSTEACS there is an emphasis on an initial approach to care of an ischemia guided strategy replacing a previous conservative management approach. However, individualizing care in patients with associated conditions and comorbidities is a critical imperative in patients with an ACS and coexisting anemia.

NSTEACS incidence is rising year on year with negligible changes in mortality and morbidity. The OPERA registry ${ }^{5}$ demonstrated that in-hospital mortality of NSTEACS patients remains high at $4.3 \%$ (STEMI $4.6 \%$ ) and at 1 year $11.6 \%$

(STEMI 9.0\%).

The interaction between anemia and NSTEACS, bleeding and death

Many risk factors for bleeding (GI sources being a significant proportion) are shared risk factors for increased cardiovascular risk in patients who have suffered a NSTEACS (renal impairment, age, diabetes, heart failure). These high risk cardiac patients often have potentially most to gain from an invasive treatment strategy but often don't receive invasive 
investigation nor treatment ${ }^{6}$. A large proportion of NSTEACS patients with high risk scores will have increased risk of bleeding.

The largest study looking at baseline anemia in NSTEACS is that by the OASIS investigators ${ }^{7}$ who retrospectively analyzed 32,170 patients from the OASIS trials. Baseline anemia (WHO criteria) was seen in $20.5 \%$ of patients overall, despite subjects with known malignancy or hemorrhagic diathesis already been excluded. Patients were assessed with the GRACE cardiovascular risk score ${ }^{8}$ and were separated into groups according to hemoglobin $(\mathrm{Hb})$ concentration (in $1 \mathrm{~g} / \mathrm{dL}$ groups from $<10$ to $>17 \mathrm{~g} / \mathrm{dL}$ ). There was a significant inverse correlation between GRACE risk score and baseline $\mathrm{Hb}$ levels. Patients with the lowest baseline Hb levels tended to be older, more frequently females, with lower body weight, higher heart rate, and lower systolic/diastolic blood pressure than patients with higher levels. They tended to have more comorbidities as shown by the more frequent history of coronary artery disease intervention], or coronary artery bypass grafting $[\mathrm{CABG}]$ ), higher rates of diabetes, hypertension, and heart failure than patients with higher levels of baseline Hb. Creatinine clearance tended to be lower in individuals with low baseline Hb levels. Renal anemia should also be carefully considered before embarking on coronary angiography and PCI as the addition of contrast induced nephropathy in NSTEACS patients has further prognostic implications in addition to the presence of anemia ${ }^{2}$.

There was a significant relationship between patients with lower levels of baseline $\mathrm{Hb}$ and reduced rates of diagnostic coronary angiography (from $62.5 \%$ in the normal $\mathrm{Hb}$ range down to $38.9 \%$ in the group with $\mathrm{Hb}$ between $10-11 \mathrm{~g} / \mathrm{dL}$ ) and PCI (from $42.9 \%$ to $20.5 \%$ normal to $10-11 \mathrm{Hb}$ range) during hospitalization. Patients with lower $\mathrm{Hb}$ tended to receive a lower frequency prescription of beta-blockers and ACE inhibitors. Interestingly clopidogrel usage did not significantly differ between the groups but aspirin usage was reduced from $98.2 \%$ to 95.2\% in those with the highest and lowest baseline $\mathrm{Hb}$ levels respectively. In a progressive manner, as $\mathrm{Hb}$ level reduces the rate of death (3.3\% to $8.9 \%)$, death/MI (6.0\% to $11.6 \%)$, and MI (2.9\% to $3.9 \%)$ all significantly increase (highest to lowest $\mathrm{Hb}$ groups in brackets). The overall frequency of major bleeding was 3.3\% with an increased frequency as the $\mathrm{Hb}$ dropped (2.2\% to $6.7 \%$ ). Multiple regression analysis shows that baseline $\mathrm{Hb}$ together with increasing age, heart failure and creatinine clearance $(\mathrm{CrCl})$ are all independent predictors of procedural and nonprocedural bleeding.

What will the impact of a major bleed have on mortality?

Major bleeds have a profound effect on the mortality of NSTEACS patients. Manoukian et al., in their analysis

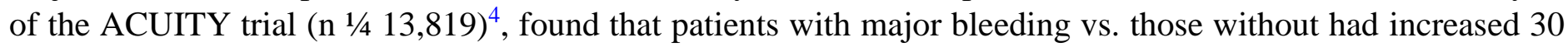
day mortality $(7.3 \%$ vs. $1.2 \%, \mathrm{p}<0.0001)$. Major bleeding was an independent predictor of 30 day mortality (OR $7.55,95 \%$ CI 4.68 to

$12.18 ; \mathrm{p}<0.0001)$, and a stronger independent predictor of mortality than MI (OR 3.96, 95\% CI 2.45-6.42; p < $0.0001)$. Ischemic events were also higher in the major bleeding group (23.1\% vs. 6.8\%; p < 0.0001). An increase in stent thrombosis in the bleeding group (3.4\% vs. $0.6 \%$; < 0.0001$)$ was also followed by an increase in MI and unplanned revascularization. These findings are reinforced in the Eikelboom et al. metaanalysis of the OASIS registry ${ }^{9}$, and the $\mathrm{CURE}^{10}$ trial with ACS patients who were predominantly medically managed with only $10.4 \%$ having PCI. The association of major bleeding with increased hazard of death in the first 30 days was evident even when adjusted for baseline differences and propensity matched (HR 5.37; 95\% CI, 3.97-7.26; $\mathrm{p}<0.0001$ ). Rates of MI and stroke were increased in a similar fashion with one in three of those dying after a major bleed having a repeat MI prior to death.

Gastrointestinal bleeding: the commonest non-access site bleeding source and its impact in NSTEACS

The commonest cause of non-access site blood loss is secondary to gastrointestinal bleeding (GIB) ${ }^{11}$. The ACUITY trial reported a rate of $4.7 \%$ of major bleeding in moderate/high risk ACS patients ${ }^{4}$. Overall GIB rates were $1.3 \%$ (48\% TIMI major bleeding), this equates to $28.6 \%$ of all non-access site major bleeds. This rate of 
GIB may be higher as $0.75 \%$ of major bleeds with a $4 \mathrm{~g} / \mathrm{dL}$ reduction in $\mathrm{Hb}$ with no overt source of bleeding were not formerly defined as GIB. A proportion of these could be non-exteriorized GIB. The ACUITY trial highlighted that both minor and major GIB were highly significant in terms of 30 day mortality $9.6 \%$ vs. $1.4 \%$, composite ischemic outcome $19.8 \%$ vs. $7.5 \%$, cardiac death $7.4 \%$ vs. $1.2 \%$ and MI $12.0 \%$ vs. $5.1 \%$ (GIB vs. no GIB respectively, all $\mathrm{p}<0.0001)$. The impact of GIB was seen out to 1 year for all causes (HR 3.97 2.64-5.99 $\mathrm{p}$ $<0.0001$ ) and cardiac causes (HR $3.772 .14-6.63 \mathrm{p}<0.0001$ ) of death together with composite ischemia (HR $1.901 .37-2.64 \mathrm{p} \mathrm{1/4}$ 0.0001). The independent predictors for GIB appear similar to those for major bleeding of all causes: baseline anemia (OR 2.05 1.42-2.97 p 1/4 0.0001), ST segment deviation >1 mm (OR 1.79 1.12-2.85 p 1/4 0.015), smoking (OR $1.731 .14-2.64$ p 1/4 0.011), age per 10 years (OR $1.611 .35-1.92 \mathrm{p}<0.0001$ ), and diabetes mellitus (OR 1.46 1.01-2.10 p 1/4 0.044).

A further adverse sequela from worsening anemia or GI hemorrhage, in the context of ACS, is the need for blood transfusion. A meta-analysis of three large clinical trials found that transfusion in the ACS setting increased mortality, despite adjustment for other predictive factors ${ }^{12}$.

\section{Calculating cardiovascular risk and bleeding risk prior to PCI}

A risk stratified application of an invasive approach (angiography followed by appropriate revascularization) to patients with NSTEACS is generally applied in the $\mathrm{UK}^{13,14}$. Moderate/ high risk patients gain short and long term benefits including reduced rates of death and MI. Risk scoring is central to applying an invasive approach to identify those that will benefit greatest ${ }^{15}$. The GRACE risk score allows a calculation of in-hospital and 6 month outcomes (death/MI) ${ }^{8}$. A low, intermediate and high GRACE score predicts a $<3 \%, 3-8 \%$ and $>8 \%$ risk of death at 6 months respectively ${ }^{8}$. A more difficult calculation is that of bleeding risk. All the major guidelines for treating NSTEACS patients urge special attention to patients with high bleeding risk but few suggest specific instructions for calculating bleeding risk.

Measuring pre-procedural bleeding risk (using only admission clinical variables) the CRUSADE score is the most suitable currently available (with a c-statistic of 0.71$)^{16}$. Based on 71,277 patients and validated with 117,857 patients it identifies eight independent baseline predictors of in-hospital major bleeding in NSTEACS patients (male/female, diabetes, prior vascular disease, heart rate, systolic BP [blood pressure], CCF [congestive cardiac failure] signs, baseline $\mathrm{HCT}$ [haematocrit] $<36 \%$ and $\mathrm{CrCl}$ ). The majority of major bleeding episodes $(60 \%)$ are nonaccess site. In a recent meta-analysis ${ }^{17}$, bleeding of any type was detrimental to mortality but in particular non-access site bleeding (unadjusted 1 year mortality of TIMI [thrombolysis in myocardial infarction] major and minor bleeds: no bleed $2.54 \%$, access site $6.16 \%$ and non-access site $14.4 \%$ ).

How common is low baseline $\mathrm{Hb}$ and how can/ should we investigate the NSTEACS patient?

Not all patients with low baseline $\mathrm{Hb}$ have a GI etiology for this. Equally not all patients who develop blood loss secondary to GI etiology have low $\mathrm{Hb}$ prior to this. Exclusion of other major causes of low $\mathrm{Hb}$ needs to be identified initially, for example anemia of chronic disease, renal impairment, hematuria, menorrhagia, hemolytic anemia, etc. A patient with either low $\mathrm{Hb}$ or high bleeding risk should initially have the risks of invasive investigation balanced against the benefits.

Anemia due to chronic blood loss will typically be associated with a low mean cell volume and usually a low ferritin level. This type of anemia is termed iron deficiency anemia (IDA) and in the vast majority the source of bleeding will be in the gastrointestinal tract. Its prevalence in adult males and post-menopausal females is 2 $5 \%{ }^{18}$. Not all cases of IDA are due to bleeding, malabsorption and blood dyscrasias such as myeloma can also present in this fashion and require hematological input and investigation (Table 1) ${ }^{19}$.

A focused history for excluding other causes of anemia, as above, is important. Ferritin and iron binding studies may assist with overt markers of iron deficiency but these may not be present if anemia is of recent onset following use of dual antiplatelet drugs and anticoagulants. Ferritin is an acute phase reactant and may be elevated 
in the presence of concurrent inflammation and mask iron deficiency in certain individuals. If iron deficiency is confirmed then all patients should have serology checked to exclude celiac disease.

Investigating the GI tract usually involves performing gastroscopy (with duodenal biopsy) and colonoscopy; the latter may be deemed unsuitable if the patient has significant comorbidity. Computed tomographic colonography (CTC) (or barium enema if unavailable) is a suitable alternative, and also indicated if colonoscopy is attempted but not completed to the cecum.

Table 1. Causes of iron deficiency anemia with prevalence as percentage of total.

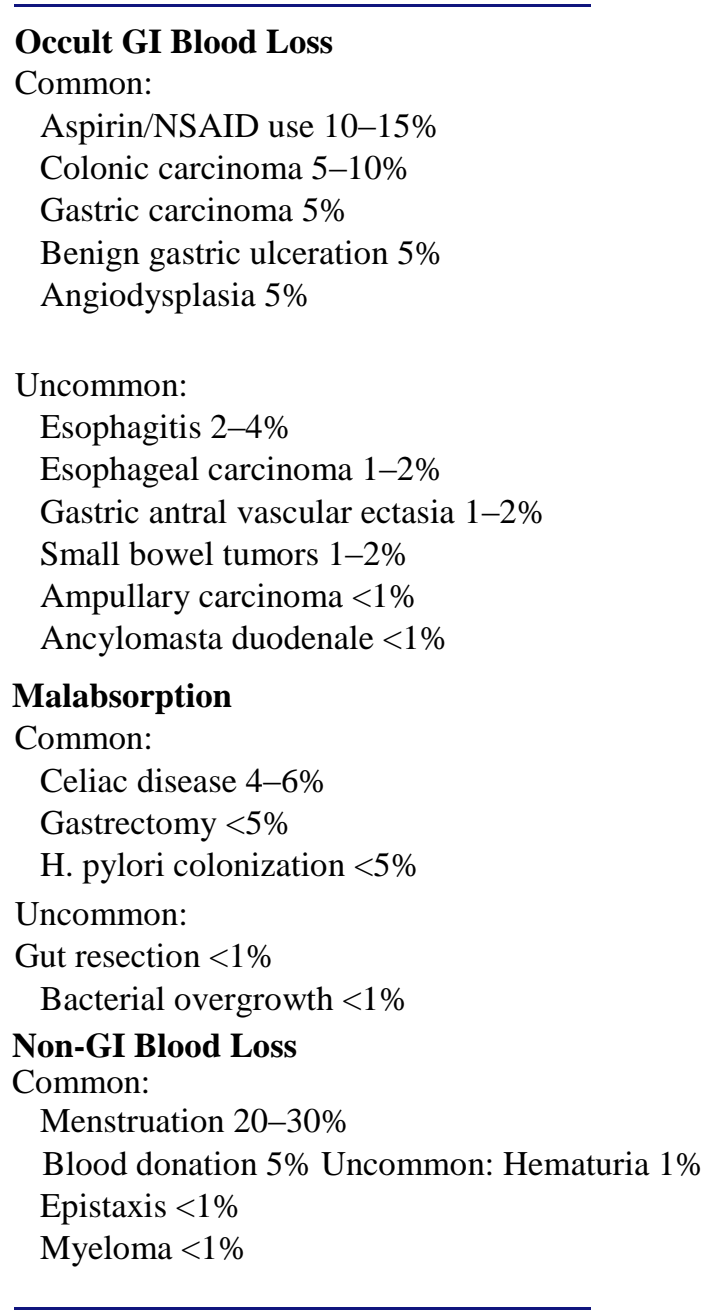

Table taken from BSG Guidelines for Management of Iron Deficiency Anemia ${ }^{21}$.

Small bowel examination can be achieved using video capsule endoscopy/enteroscopy; it is not routinely recommended unless there are symptoms to suggest small bowel pathology or $\mathrm{Hb}$ levels cannot be maintained with iron therapy ${ }^{20}$. The overall diagnostic yield for investigating IDA ranges from $49 \%$ to $75 \% 22,23$.

\section{Risk of upper GI endoscopy in NSTEACS}

It remains common clinical practice to postpone endoscopy until at least 6 weeks after NSTEACS to "enable the cardiac condition to stabilise". This area has scant evidence to support what are arbitrary time cut-offs. The 6 week interval is concordant with surgical practice of delaying elective noncardiac surgery after myocardial infarction (MI) ${ }^{24}$. Whilst the function of these delays is to try and address the risk of investigation (GI) vs. potential benefit it remains questionable to globally apply these protocols in light of the limited evidence available. 
A case-control study of 200 patients who underwent gastroscopy within 30 days of a MI found that $7.5 \%$ had complications vs. $1.5 \%$ of controls $(p<0.007)$; two of these complications were severe ${ }^{25}$. The indication for OGD [Oesophagogastroduodenoscopy] in the majority of cases was acute GI bleeding (65\%) and MI was defined as any CK [Creatinine Kinase] level > $225 \mathrm{U} / \mathrm{L}$ and a CK-MB [Creatinine Kinase Muscle bound] fraction $>5 \%$. This 1999 analysis was prior to the widespread use of thienopyridines and using MI definitions, which included mostly STEMI patients with larger MIs. The two severe complications included a fatal ventricular tachycardia and near respiratory arrest requiring intubation.

The 13 mild complications included 11 episodes of transient hypotension and two of transient hypoxia. Control group complications were all mild.

Another study of 85 patients who underwent gastroscopy within 28 days of MI had a complication rate of $8 \%$. All of these were mild (transient hypotension, hypoxia or bradycardia) with no procedure related mortality ${ }^{26}$. A retrospective study of a variety of endoscopic procedures performed within 30 days of MI analyzed the safety of the specific timing of endoscopy (number of days post-MI), and with respect to the severity of cardiac damage ${ }^{21}$. In 135 patients, major complications occurred in 1.5\%; episodes occurred when endoscopy was performed on the same day as MI. The severity of MI (according to presence of STEMI, left ventricular impairment, and troponin peak) had no significant effect.

In a prospective study of emergency gastroscopy performed for upper GI bleeding in 50 patients with no coronary artery disease (CAD) compared to 50 with CAD there was a higher incidence of ventricular arrhythmia, and silent ischemia in those with known CAD, although most were subclinical. The only in-hospital cardiac death was a patient in the CAD group with three vessel disease who developed angina 5 days after gastroscopy and died of ventricular fibrillation, arguably unrelated to the gastroscopy ${ }^{21}$. ECG monitoring of patients with stable angina undergoing gastroscopy has showed evidence of silent ischemia in $42 \%$ of patients. The main risk factor appeared to be the presence of tachycardia, and so the use of b-blockers and Ivabradine was recommended with Buscopan avoidance ${ }^{27}$.

\section{Risk of lower GI endoscopy in NSTEACS}

Data on performing colonoscopy in NSTEACS is sparse. Colonoscopy is regarded as being more physiologically demanding than upper GI endoscopy in part because of electrolyte and fluid shifts secondary to bowel preparations resulting in hypotension but also vagal nerve stimulation due to mesenteric stretch and air insufflation. These complications can be reduced with optimal colonoscopy technique. In a case-control study 100 patients had colonoscopy within 30 days of MI. The complication rate (9\%) was significantly higher than controls $(1 \%),(\mathrm{p}<0.03)^{28}$. Complications were mostly non-significant minor ones such as transient hypotension and bradycardia, with one major complication involving death of a patient 14 hours after colonoscopy (thought unlikely to be related to the procedure). Colonoscopy completion rate was $85 \%$ in study patients vs. $93 \%$ in controls.

Another study of patients undergoing elective colonoscopy indicated more cardiac arrhythmias in patients with stable angina ${ }^{29}$. Importantly this did not equate to any clinical features at the time of the procedure. Two deaths occurred in the study, both in the Ischaemic heart disease (IHD) group (4.6\% 30 day mortality in this group). Flexible sigmoidoscopy was considered to be safe within 30 days of MI, complications rare (2.6\%) and minor ${ }^{30}$.

\section{Risk of endoscopic GI investigations: in summary}

The studies outlined for both endoscopic modalities are limited by size, case selection and measurement bias, their retrospective nature and lack of applicability to NSTEACS patients where overall size of MI is often smaller is questionable. In the context of NSTEACS patients with moderate, high and highest GRACE risk (mortality risk: $3-6 \%,>6-9 \%$ and $>9 \%$ at 6 months respectively) this data suggests that a proportion of these patients may benefit from tailored cardiovascular and GI investigation despite their anemia when balanced against the risk of 
investigating it acutely. Further large scale prospective studies are required in NSTEACS patients undergoing GI investigation and treatment for contemporary safety data.

Alternative modes of investigation

Radiological investigation of the upper GI tract is of limited value and is not recommended in the BSG guidelines for iron deficiency anemia management. Barium meals and CT scans can only detect large structural abnormalities and are considered to be inferior to an OGD to visualize the upper GI tract.

Radiological imaging offers a less invasive approach for investigating the lower GI tract and may have benefits in the setting of NSTEACS. Furthermore colonoscopy will not reach the cecum in $8 \%$ of cases, thereby failing to exclude proximal colonic pathology ${ }^{31}$. CTC is superior to barium enema (BE) in detecting colorectal cancer and large colonic polyps, and has a similar sensitivity to colonoscopy ${ }^{32,33}$. A meta-analysis showed that for polyps $>1 \mathrm{~cm}$, BE had a sensitivity of $48 \%$ and specificity of $90 \%$, whilst CTC's sensitivity was $81 \%$ and specificity $96 \%{ }^{34}$. Both techniques can be limited by the need for a full bowel preparation.

An alternative radiological investigation which does not involve bowel preparation or air insufflation is minimal preparation CT. This uses oral and intravenous contrast and has been used in frail patients in whom more invasive tests are considered inappropriate. Although not effective at detecting small polyp lesions, it does have sensitivity for detecting colorectal cancers (sensitivity of $83 \%$ and specificity of $90 \%)^{35}$. In the context of NSTEACS the detection of colorectal cancer rather than small polyps is of greater significance, and so this less invasive investigation is useful. In obscure GI bleeding capsule endoscopy has a diagnostic yield of $63 \%{ }^{36}$. If it becomes more widely accepted it could be a valuable tool for investigating IDA in the setting of NSTEACS.

Performing both cardiac and GI investigations: revascularization strategies directed by GI pathology

Performing coronary angiography allows anatomic risk stratification delineating minor, moderate or major coronary disease and allows further evaluation of high risk appearances such as thrombus burden/ulcerated plaque morphology, etc. These appearances can dictate and potentially predict future cardiac events.

The angiographic appearances also dictate the mode of revascularization required such as coronary artery bypass grafting $(\mathrm{CABG})$ or PCI. Peri-procedural adjunctive pharmacotherapy can be adjusted towards a lower bleeding risk strategy by reduction in the use of glycoprotein II/IIIa inhibitors in 


\section{Anemia and Non-ST Elevation ACS - Double Jeopardy Suggested decision making tree}

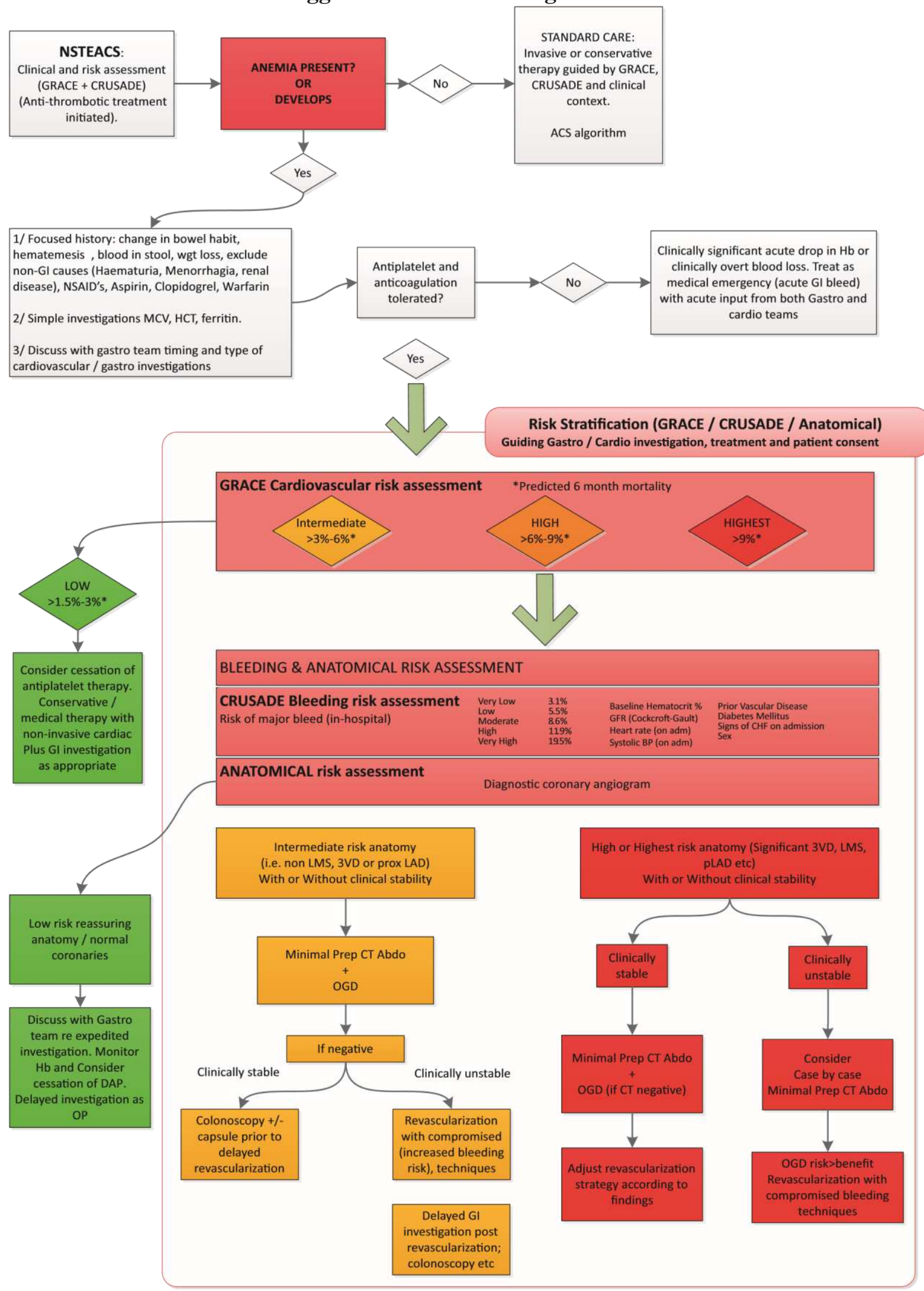

Figure 1. Anemia and non-ST-elevation ACS - double jeopardy. Suggested decision making tree. 
those with high risk features. Antiplatelet therapies can also be modified away from more potent drugs (ticagrelor and prasugrel) depending on the GI pathology found. PCI treatments can also be modified, such as balloon only angioplasty, bare metal and drug eluting stent implantation to decreased long term bleeding risk due to dual antiplatelet treatment duration. Knowledge of incipient GI pathology also has clear treatment ramifications for $\mathrm{CABG}$ as the presence of pre-existing gastric or duodenal ulceration being exacerbated by heparinization and/or stress ulceration induced at surgery could materially affect outcome.

This approach (outlined in Figure 1) of diagnostic coronary angiography initially followed by GI investigations allows a crucial better informed synthesis of decision making based upon the two concurrent clinical risks posed to the patient by two distinct clinical pathologies: an acute coronary syndrome and underlying anemia of uncertain etiology. Ultimately the best possible knowledge of both pathological processes allows "optimal" decision making and thus the most appropriate guided therapy or therapies.

\section{Conclusion}

Patients presenting with an acute coronary syndrome and concomitant anemia or blood loss once they have commenced powerful antithrombotic therapy are currently inadequately risk stratified and treated. This review highlights the patients' high risk profile from a cardiac and gastroenterological perspective. The schematic presented offers a clear pragmatic clinical pathway of cardiac and GI risk stratification measures readily available on admission, variables which can enable clinicians to prioritize investigations to minimize both investigational and treatment delays and enact the most appropriate therapies based upon the distinct pathologies present.

\section{Transparency}

Declaration of funding

This paper was not funded.

Declaration of financial/other relationships

P.F., J.B., J.G., and R.A. have disclosed that they have no significant relationships with or financial interests in any commercial companies related to this study or article.

CMRO peer reviewers on this manuscript have no relevant financial or other relationships to disclose.

\section{Acknowledgments}

Dr R Anderson and Dr P Freeman were recipients of a project grant by the British Heart Foundation charity. 


\section{References}

1. Foley P, Foley S, Kinnaird T, Anderson R. Clinical review: gastrointestinal bleeding after percutaneous coronary intervention: a deadly combination. QJM 2008;101:425

2. Kitai Y, Ozasa N, Morimoto T, et al. Prognostic implications of anemia with or without chronic kidney disease in patients undergoing elective percutaneous coronary intervention. Int J Cardiol 2013;168:5221-8

3. Anand I, McMurray JJ, Whitmore J, et al. Anemia and its relationship to clinical outcome in heart failure. Circulation 2004;110:149-54

4. Manoukian SV, Feit F, Mehran R, et al. Impact of major bleeding on 30-day mortality and clinical outcomes in patients with acute coronary syndromes: an analysis from the ACUITY trial. J Am Coll Cardiol 2007;49:1362-8

5. Montalescot G, Dallongeville J, Van Belle E, et al. STEMI and NSTEMI: are they so different? 1 year outcomes in acute myocardial infarction as defined by the ESC/ACC definition (the OPERA registry). Eur Heart J 2007;28:1409-17

6. Fox KAA, Anderson FA, Dabbous OH, et al. Intervention in acute coronary syndromes: do patients undergo intervention on the basis of their risk characteristics? The Global Registry of Acute Coronary Events (GRACE). Heart 2007;93:177-82

7. Bassand J-P, Afzal R, Eikelboom J, et al. Relationship between baseline haemoglobin and major bleeding complications in acute coronary syndromes. Eur Heart J 2010;31:50-8

8. Fox KAA, Dabbous OH, Goldberg RJ, et al. Prediction of risk of death and myocardial infarction in the six months after presentation with acute coronary syndrome: prospective multinational observational study (GRACE). BMJ 2006;333:1091.

9. Eikelboom JW, Mehta SR, Anand SS, et al. Adverse impact of bleeding on prognosis in patients with acute coronary syndromes.

Circulation 2006;114:774-82

10. Yusuf S, Bijsterveld N, Moons A. Effects of clopidogrel in addition to aspirin in patients with acute coronary syndromes without ST-segment elevation: the Clopidogrel in Unstable Angina to Prevent Recurrent Events Trial Investigators. N Engl J Med

2001;345:494-502

11. Nikolsky E, Stone GW, Kirtane AJ, et al. Gastrointestinal bleeding in patients with acute coronary syndromes: incidence, predictors, and clinical implications: analysis from the ACUITY (Acute Catheterization and Urgent Intervention Triage Strategy) trial. J Am Coll Cardiol 2009;54:1293-302

12. Rao SV, Jollis JG, Harrington RA, et al. Relationship of blood transfusion and clinical outcomes in patients with acute coronary syndromes. JAMA 2004;292:1555-62

13. Mehta SR, Cannon CP, Fox KAA, et al. Routine vs selective invasive strategies in patients with acute coronary syndromes: a collaborative meta-analysis of randomized trials. JAMA 2005;293:2908-17

14. Fox KAA, Poole-Wilson P, Clayton TC, et al. 5-year outcome of an interventional strategy in non-STelevation acute coronary syndrome: the British Heart Foundation RITA 3 randomised trial. Lancet 2005;366:914-20

15. The National Clinical Guideline Centre. Unstable Angina and NSTEMI: the Early Management of Unstable Angina and Non-STSegment-Elevation Myocardial Infarction. 2010

16. Subherwal S, Bach RG, Chen AY, et al. Baseline risk of major bleeding in non-ST-segment-elevation myocardial infarction: the CRUSADE (Can Rapid risk stratification of Unstable angina patients Suppress ADverse outcomes with Early implementation of the ACC/AHA guidelines) bleeding score. Circulation 2009;119: 1873.19 
17. Verheugt FWA, Steinhubl SR, Hamon M, et al. Incidence, prognostic impact, and influence of antithrombotic therapy on access and nonaccess site bleeding in percutaneous coronary intervention. JACC: Cardiovascular Interventions 2011;4:191-7

18. Calvey HD, Castleden CM. Gastrointestinal investigations for anaemia in the elderly: a prospective study. Age Ageing 1987;16: 399-404

19. Goddard AF, James MW, McIntyre AS, Scott BB. Guidelines for the management of iron deficiency anaemia. Gut 2011;60:1309-16

20. Hershko C, Vitells A, Braverman DZ. Causes of iron deficiency anemia in an adult inpatient population. Effect of diagnostic workup on etiologic distribution. Blut 1984;49:347-52

21. Spier BJ, Said A, Moncher K, Pfau PR. Safety of endoscopy after myocardial infarction based on cardiovascular risk categories: a retrospective analysis of 135 patients at a tertiary referral medical center. J Clin Gastroenterol 2007;41:462-7

22. Gordon SR, Smith RE, Power GC. The role of endoscopy in the evaluation of iron deficiency anemia in patients over the age of 50. Am J Gastroenterol 1994;89:1963-7

23. Willoughby JM, Laitner SM. Audit of the investigation of iron deficiency anaemia in a district general hospital, with sample guidelines for future practice. Postgrad Med J 2000;76:218-22

24. Poldermans D, Bax JJ, Boersma E, et al. Guidelines for pre-operative cardiac risk assessment and perioperative cardiac management in non-cardiac surgery: the Task Force for Preoperative Cardiac Risk Assessment and Perioperative Cardiac Management in Non-cardiac Surgery of the European Society of Cardiology (ESC) and endorsed by the European Society of Anaesthesiology (ESA). Eur J

Anaesthesiol 2010;27:92-137

25. Cappell MS, Iacovone FM Jr. Safety and efficacy of esophagogastroduodenoscopy after myocardial infarction. Am J Med 1999;106:29-35

26. Mumtaz K, Ismail FW, Jafri W, et al. Safety and utility of oesophago-gastro-duodenoscopy in acute myocardial infarction. Eur J Gastroenterol Hepatol 2008;20:51-5

27. Tseng PH, Liou JM, Lee YC, et al. Emergency endoscopy for upper gastrointestinal bleeding in patients with coronary artery disease. Am J Emerg Med 2009;27:802-9

28. Schenck J, Muller CH, Lubbers H, et al. Does gastroscopy induce myocardial ischemia in patients with coronary heart disease? Endoscopy 2000;32:373-6

29. Cappell MS. Safety and efficacy of colonoscopy after myocardial infarction: an analysis of 100 study patients and 100 control patients at two tertiary cardiac referral hospitals. Gastrointest Endosc 2004;60:9019

30. George AT, Davis C, Rangaraj A, et al. Cardiac ischaemia and rhythm disturbances during elective colonoscopy. Frontline Gastroenterology 2010;1:131-7

31. Cappell MS. Risks versus benefits of flexible sigmoidoscopy after myocardial infarction: an analysis of 78 patients at three medical centers. Am J Med 2004;116:707-10

32. Gavin DR, Valori RM, Anderson JT, et al. The national colonoscopy audit: a nationwide assessment of the quality and safety of colonoscopy in the UK. Gut 2013;62:242-9

33. Atkin W, Dadswell E, Wooldrage K, et al. Computed tomographic colonography versus colonoscopy for investigation of patients with symptoms suggestive of colorectal cancer (SIGGAR): a multicentre randomised trial. Lancet

2013;381:1194-202

34. Halligan S, Wooldrage K, Dadswell E, et al. Computed tomographic colonography versus barium enema for diagnosis of colorectal cancer or large polyps in symptomatic patients (SIGGAR): a multicentre randomised trial. Lancet 2013;381:1185-93

35. Koo BC, Ng CS, J UK-I, et al. Minimal preparation CT for the diagnosis of suspected colorectal cancer in the frail and elderly patient. Clin Radiol 2006;61:127-39 
36. Sidhu R, Sanders DS, Morris AJ, McAlindon ME. Guidelines on small bowel enteroscopy and capsule endoscopy in adults. Gut

2008;57:125-36 